This is a post-peer-review, pre-copyedit version of:

Guidi F., Guerra A., Clemente A., Dardari D., D’Errico R. (2016) Energy Detection Performance with Massive Arrays for Personal Radars Applications. In: Noguet D., Moessner K., Palicot J. (eds) Cognitive Radio Oriented Wireless Networks. CrownCom 2016. Lecture Notes of the Institute for Computer Sciences, Social Informatics and Telecommunications Engineering, vol 172. Springer, Cham

The final authenticated version is available online at:

https://doi.org/10.1007/978-3-319-40352-6_52

This version is subjected to Springer Nature terms for reuse that can be found at: https://www.springer.com/gp/openaccess/authors-rights/aam-terms-v1

C ICST Institute for Computer Sciences, Social Informatics and Telecommunications Engineering 2016 


\title{
Energy Detection Performance with Massive Arrays for Personal Radars Applications
}

\author{
Francesco Guidi ${ }^{1,2}$, Anna Guerra ${ }^{3}$, Antonio Clemente ${ }^{1,2}$, Davide Dardari ${ }^{3}$, and \\ Raffaele D'Errico ${ }^{1,2}$ \\ 1 CEA, LETI, MINATEC Campus, 38054 Grenoble, France, \\ \{francesco.guidi, antonio.clemente, raffaele.derrico\}@cea.fr, \\ ${ }^{2}$ Univ. Grenoble-Alpes, 38000 Grenoble, France \\ 3 DEI, University of Bologna,Via Venezia 52, 47521 Cesena, ITALY, \\ \{anna.guerra3, davide.dardari\}@unibo.it).
}

\begin{abstract}
The idea to adopt massive arrays for personal radars applications is facing a rapid growth, thanks to the high scanning resolution achievable with the large number of antennas employed. In fact, such multi-antenna systems enable the possibility to detect and localize surrounding objects through an accurate beamforming procedure. In this paper we show a classical energy-detection approach for target ranging and localization, where the threshold is designed according to the receiver noise only, since an ideal laser-beam antenna is considered. Successively, we show the ambiguities that could arise when the presence of side-lobes cannot be neglected (e.g., when considering real massive arrays instead of ideal pencil-beam like radiation patterns) and we propose a set of guidelines that can be followed from a system design point-of-view to overcome this issue.
\end{abstract}

Key words: Massive arrays, personal radar, target detection, side-lobes.

\section{Introduction}

The adoption of massive arrays is facing a rapid growth in several ranging and localization applications, such as personal radars [1], thanks to the possibility to achieve a precise and high-scanning resolution given by the large number of adopted antennas [2].

The concept of personal radar has been recently proposed in $[1,3]$ where it has been shown the possibility to jointly use millimeter-waves ( $\mathrm{mmW})$ and wideband massive arrays technologies for indoor environment mapping. Thanks to this technology, it is possible to avoid the adoption of a dedicated very highdirectional antenna with mechanical steering, as proposed in [4-6], which can not be easily integrated into portable radar devices. The consequent near-pencil beam of massive arrays returns a precise angle and range information thus making the modeling and characterization of the environment with personal radars very similar to that based on laser. 
Different literature has been produced for the analysis of the localization performance of wideband large antenna arrays. In fact, wideband signals are the best candidate to achieve high ranging performance [7], but a strict phase control in beamforming, i.e. the adoption of precise and costly phase shifters and delay lines, becomes necessary to assure a perfect signal alignment. A cheaper and alternative solution is to adopt digitally controlled phase shifters implementing a discrete set of phase shifts at the price of a reduced signal alignment and an increased level of side-lobes $[8,9]$. Despite the high-ranging accuracy which can be achieved by the adoption of such systems, all these effects have to be accounted for when target detection is performed for different steering directions.

In this paper we propose a low-complexity non-coherent detection scheme, where the detection of objects is performed by a massive array which steers its beam in different directions in order to detect and localize objects. Thanks to the near-pencil beam array considered, all the measured contributions are associated to the considered steering direction. According to the receiver performance, we describe a set of guidelines to be followed when energy detection with massive arrays is performed.

The rest of the paper is organized as follows. In Sec. 2 we first show the threshold design, and successively we evaluate the side-lobe effects when the previously defined threshold based on receiver noise is adopted. Finally, in Sec. 3 we report a case study where real massive arrays are considered, and we discuss a possible solution to overcome the issue when energy detection is performed.

\section{Target Detection Scheme}

The personal radar concept is based on the idea that the surrounding objects are detected and localized thanks to the beamforming procedure enabled by massive arrays.

The system herein considered exploits monostatic scattering, i.e. the transmitter and receiver are co-located. For each steering direction $\theta_{b}$, the massive array steers its main beam towards that direction, and collects the overall backscattered response in order to detect and localize objects. To our purpose, first we account for a laser-like antenna, with a radiation pattern that permits to neglect the side-lobes effect, and thus, we adopt a classical constant false alarm rate (CFAR) approach accounting for the receiver noise only. Second, we focus our analysis to real antennas in which side lobes might cause false target detection. In fact, the presence of a target could be detected and assigned in a certain direction even if it is not effectively located in that part, as shown in Fig. 1. In addition, they could even cause errors in the ranging procedure, i.e. the distance of target 1 and 2 of Fig. 1 can be confused. Thus, once the threshold has been set, we theoretically evaluate the impact of real massive arrays on the detection performance. For our specific case, we consider massive arrays at $60 \mathrm{GHz}$ which can be considered a natural candidate for personal radars applications due to their radiation characteristics. 
In the following we describe the receiver scheme considered, and the threshold design by accounting for an ideal laser-beam antenna.

\subsection{Receiver Scheme}

The detection scheme we propose is a non-coherent approach based on energy detection to account for the complete uncertainty we have on the received waveform shape.

For each steering direction $\theta_{b}$, define the received signal as

$$
r\left(t, \theta_{b}\right)=s\left(t, \theta_{b}\right)+n(t) .
$$

with $n(t)$ indicating the noise term and $s\left(t, \theta_{b}\right)$ the received waveform including all the signals coming from the steering direction $\theta_{b}$.

The received signal is first passed through an ideal bandpass filter with center frequency $f_{\mathrm{c}}$ to eliminate out-of-band noise. ${ }^{1}$ The filtered signal is denoted by

$$
y\left(t, \theta_{b}\right)=x\left(t, \theta_{b}\right)+z(t)
$$

where $x\left(t, \theta_{b}\right)=s\left(t, \theta_{b}\right) \otimes h_{\mathrm{F}}(t)$ and $z(t)=n(t) \otimes h_{\mathrm{F}}(t)$ with $h_{\mathrm{F}}(t)$ being the impulse response of the filter.

Energy evaluations are performed over time interval $T_{\mathrm{ED}}$, with $N_{\mathrm{bin}}=$ $\left\lfloor T_{\mathrm{f}} / T_{\mathrm{ED}}\right\rfloor$ representing the number of integration bins each time frame $T_{\mathrm{f}}$ is divided in, that is

$$
e_{m}\left(\theta_{b}\right)=\int_{(m-1) T_{\mathrm{ED}}}^{m T_{\mathrm{ED}}}\left[y\left(t, \theta_{b}\right)\right]^{2} d t .
$$

with $m=1, \ldots, N_{\text {bin }}$. The detection strategy consists in comparing each element $e_{m}\left(\theta_{b}\right)$ with a threshold $\xi_{m}$. If the energy value of at least one bin is above the threshold, then the target is detected and it is assumed present in the steering direction $\theta=\theta_{b}$.

We define the following two figures of merit: (i) the probability of false alarm (PFA) $P_{\mathrm{FA}}$ as the probability of deciding that a target is detected, when it is not effectively within the considered scenario, due to presence of receiver noise; (ii) the crossing probability $P_{\mathrm{c}}$ as the probability that the threshold is overcome due to the signal backscattered from a target placed in the scenario.

If the threshold is exceeded for $m=\hat{m}$, the coordinate $\hat{m}$ leads to an estimate of the target time-of-arrival (TOA) and, jointly with the steering direction $\theta_{b}$, it provides the spatial position of the target in surrounding environment. Consider now the elements $e_{m}\left(\theta_{b}\right)$ of the decision energy bins. The presented decision rule consists in

$$
\text { Decide }:\left\{\begin{array}{lll}
\widehat{\mathcal{H}}_{0}, & \text { if } e_{m}\left(\theta_{b}\right)<\xi_{m} & \forall\{m\}, \\
\widehat{\mathcal{H}}_{1}, & \text { if } \exists\{m\} \quad \text { s.t. } & e_{m}\left(\theta_{b}\right) \geq \xi_{m} .
\end{array}\right.
$$

Define now, for each energy bin, the normalized energy detector test

\footnotetext{
${ }^{1}$ This operation is necessary since the receiver is energy-based.
} 


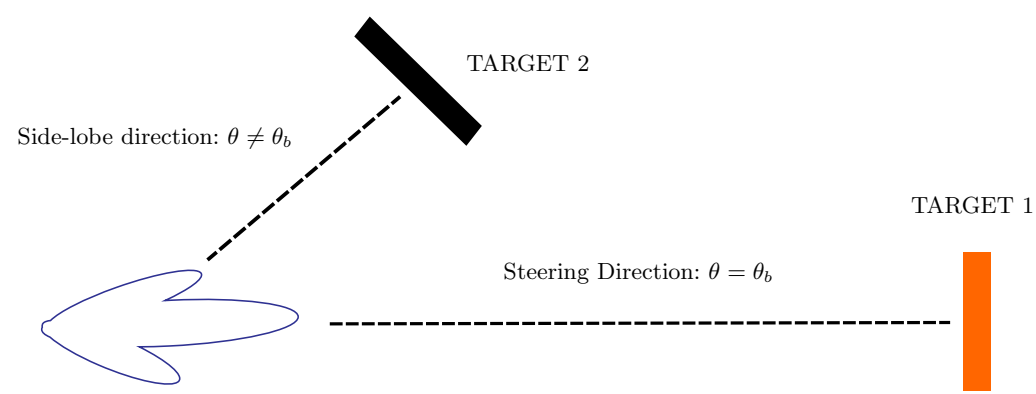

Fig. 1. Considered scenario, where for a steering direction $\theta_{b}$, the signal reflected from a target in the side-lobe direction is collected.

$$
\Lambda_{m}\left(\theta_{b}\right)=\frac{2}{N_{0}} e_{m}\left(\theta_{b}\right) \underset{\hat{\mathcal{H}}_{0}}{\stackrel{\widehat{\mathcal{H}}_{1}}{\gtrless}} \tilde{\xi}_{m}
$$

where $\tilde{\xi}_{m}=\frac{N_{0}}{2} \xi_{m}$. According to [10] we have

$$
\Lambda_{m}\left(\theta_{b}\right)=\frac{2}{N_{0}} \int_{(m-1) T_{\mathrm{ED}}}^{m T_{\mathrm{ED}}}\left[y\left(t, \theta_{b}\right)\right]^{2} d t \simeq \frac{1}{\sigma^{2}} \sum_{i=(m-1) N}^{m N}\left[y_{i}\left(\theta_{b}\right)\right]^{2}
$$

where $N=2 W T_{\mathrm{ED}}, \sigma^{2}=N_{0} W$ is the noise variance, and $y_{i}$ are the sampling expansion coefficient of the equivalent low-pass (ELP) of $y(t)$ [10], taken at Nyquist rate $W$ in each interval $T_{\mathrm{ED}}$.

\subsection{Threshold evaluation criteria with ideal pencil-beam pattern}

When an ideal pencil-beam antenna is considered, we aim to preserve that the PFA due to the receiver noise does not exceed a certain value. Thus, in the presence of only the noise, i.e. $y\left(t, \theta_{b}\right)=z(t)$, eq. (5) can be written as

$$
\Lambda_{m}\left(\theta_{b}\right)=\frac{2}{N_{0}} \int_{(m-1) T_{\mathrm{ED}}}^{m T_{\mathrm{ED}}}[z(t)]^{2} d t \simeq \frac{1}{\sigma^{2}} \sum_{i=(m-1) N}^{m N}\left(z_{i}\right)^{2} .
$$

In order to set the threshold, it is well known that the output of the energy detector is distributed according to a central Chi-square distribution, with probability density function (PDF)

$$
f_{\mathrm{C}}(\alpha, \beta)=\frac{\alpha^{\left(\frac{\beta}{2}-1\right)}}{2^{\frac{\beta}{2}} \Gamma\left(\frac{\beta}{2}\right)} e^{-\frac{\alpha}{2}}, \quad \alpha \geq 0
$$

where $\Gamma(\cdot)$ is the gamma function $[11$, p. 255] and $\beta$ is the number of degrees of freedom. 


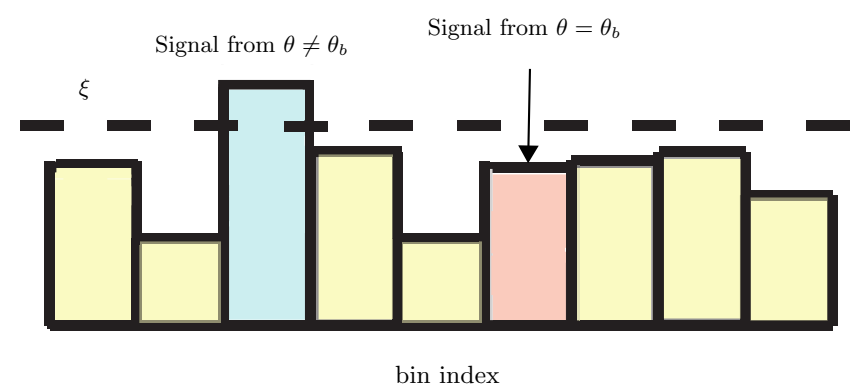

Fig. 2. Example of energy detector output, where energy-bins are compared with the threshold and might cause errors in the localization procedure.

Considering (8), a threshold-crossing event at the $m$ th bin, that is, $\Lambda_{m}\left(\theta_{b}\right)>$ $\tilde{\xi}_{m}$, results in a single-bin $p_{\mathrm{FA}}^{(m, b)}$ given by [12]

$$
p_{\mathrm{FA}}^{(m, b)}=\tilde{\Gamma}\left(\frac{N}{2}, \frac{\tilde{\xi}_{m}}{2}\right)
$$

with $\tilde{\Gamma}$ denoting the regularized Gamma function [13].

To properly set the threshold, the joint false alarms for all bins have to be taken into account. Thus, for a considered steering direction $\theta_{b}$, the overall desired false alarm probability is given by

$$
P_{\mathrm{FA}}^{\star}=1-\prod_{m=1}^{N_{\mathrm{bins}}}\left(1-p_{\mathrm{FA}}^{(m, b)}\right) \approx N_{\mathrm{bins}} \cdot p_{\mathrm{FA}}
$$

where we have assumed that all bins are statistically independent and $p_{\mathrm{FA}}^{(m, b)}=$ $p_{\text {FA }} \forall m$, and consequently we can set a desired $p_{\text {FA }}^{\star}$ per bin as

$$
p_{\mathrm{FA}}^{\star} \approx \frac{P_{\mathrm{FA}}^{\star}}{N_{\mathrm{bins}}} .
$$

Finally we can write

$$
\tilde{\xi}=2\left[\operatorname{Inv} \tilde{\Gamma}\left(\frac{N}{2}, \frac{P_{\mathrm{FA}}^{\star}}{N_{\text {bins }}}\right)\right]
$$

where $\operatorname{Inv} \tilde{\Gamma}(\cdot, \cdot)$ is the inverse gamma regularized function. Note that with such approach, the threshold does not depend on the bin index, and it is set to keep the PFA due to the receiver noise to a desired value $P_{\mathrm{FA}}^{\star}$.

\subsection{Side-Lobes Effects in Energy Detection Schemes}

The previous threshold has been set according to a central Chi-square distribution, having accounted for the presence of the noise receiver only. On the 
contrary, during the steering procedure, in real scenarios we associate all the contributions deriving from the antenna pattern to that of the steering direction $\theta_{b}$.

This approximation is often incorrect, especially when real arrays are adopted, as shown in Fig.2. Thus, in order to evaluate the impact of the side-lobes in the target detection performance, we evaluate the probability that the threshold is crossed due to the presence of a target in the side-lobe direction. In particular, define $x\left(t, \theta_{b}\right)=x_{\mathrm{sl}}\left(t, \theta_{b}\right)$ the received backscattering response under the assumption that no target is in the steering direction $\theta_{b}$, i.e. the target 1 of Fig. 1 is not present. The new random variable (RV) is now distributed as a non-central Chi-square distribution

$$
f_{\mathrm{NC}}(\alpha, \lambda, \beta)=\frac{1}{2} e^{-\frac{\alpha+\lambda}{2}}\left(\frac{\alpha}{\lambda}\right)^{\frac{\beta-2}{4}} I_{\frac{\beta}{2}-1}(\sqrt{\alpha \lambda}), \quad \alpha \geq 0
$$

where $I_{\kappa}(\cdot)$ denotes the $\kappa$ th order modified Bessel function of the first kind [11, p. $374]$ and $\mathrm{PDF} f_{\mathrm{NC}}(\alpha, \lambda, \beta)[10]$, with $\beta$ being the number of degrees of freedom and $\lambda$ the non-centrality parameter (NCP).

Due to the presence of signals coming from side-lobes direction, the normalized decision variable results into

$$
\Lambda_{m}\left(\theta_{b}\right)=\frac{2}{N_{0}} \int_{(m-1) T_{\mathrm{ED}}}^{m T_{\mathrm{ED}}}\left[x_{\mathrm{Sl}}\left(t, \theta_{b}\right)+z(t)\right]^{2} d t \simeq \frac{1}{\sigma^{2}} \sum_{i=(m-1) N}^{m N}\left(x_{\mathrm{sl} i}\left(\theta_{b}\right)+z_{i}\right)^{2}
$$

where $x_{\mathrm{sl} i}\left(\theta_{b}\right)$ are the sampling expansion coefficients of the ELP of $x_{\mathrm{sl}}\left(t, \theta_{b}\right)$ [10]. In particular, the presence of $x_{\mathrm{sl} i}\left(\theta_{b}\right)$ leads to the NCP $\lambda_{m}\left(\theta_{b}\right)=2 \gamma_{m}\left(\theta_{b}\right)[10,14]$ , where $\gamma_{m}\left(\theta_{b}\right)$ is the side-lobe-level-to-noise ratio (SLLNR) per bin, given by

$$
\gamma_{m}\left(\theta_{b}\right)=\frac{1}{N_{0}} \int_{(m-1) T_{\mathrm{ED}}}^{m T_{\mathrm{ED}}} x_{\mathrm{sl}}\left(t, \theta_{b}\right)^{2} d t \simeq \frac{1}{2 \sigma^{2}} \sum_{i=(m-1) N}^{m N}\left[x_{\mathrm{sl} i}\left(\theta_{b}\right)\right]^{2} .
$$

A threshold-crossing event at the $m$ th bin, that is, $\Lambda_{m}\left(\theta_{b}\right)>\tilde{\xi}_{m}$, results in a single-bin $p_{c}^{(m, b)}$ given by [12]

$$
p_{c}^{(m, b)}=Q_{h}\left(\sqrt{\lambda_{m}\left(\theta_{b}\right)}, \sqrt{\tilde{\xi}_{m}}\right)
$$

with $Q_{h}(\alpha, \beta)=\int_{\beta}^{\infty} x\left(\frac{x}{\alpha}\right)^{k-1} \exp \left(-\frac{x^{2}+\alpha^{2}}{2}\right) I_{k-1}(\alpha x) d x$ denoting the generalized Marcum's $Q$ function of order $h=\beta / 2$ [13].

Since the signals components deriving from side-lobes direction are undesired, we aim that the threshold is not exceeded due to such signals, i.e. $p_{c}^{(m, b)} \leq p_{\mathrm{FA}}^{\star}$. If it is not the case, target detection and ranging could be wrongly performed. In the following, the impact of real antenna patterns on the detection performance is investigated, and possible solutions to counteract such effect are reported. 


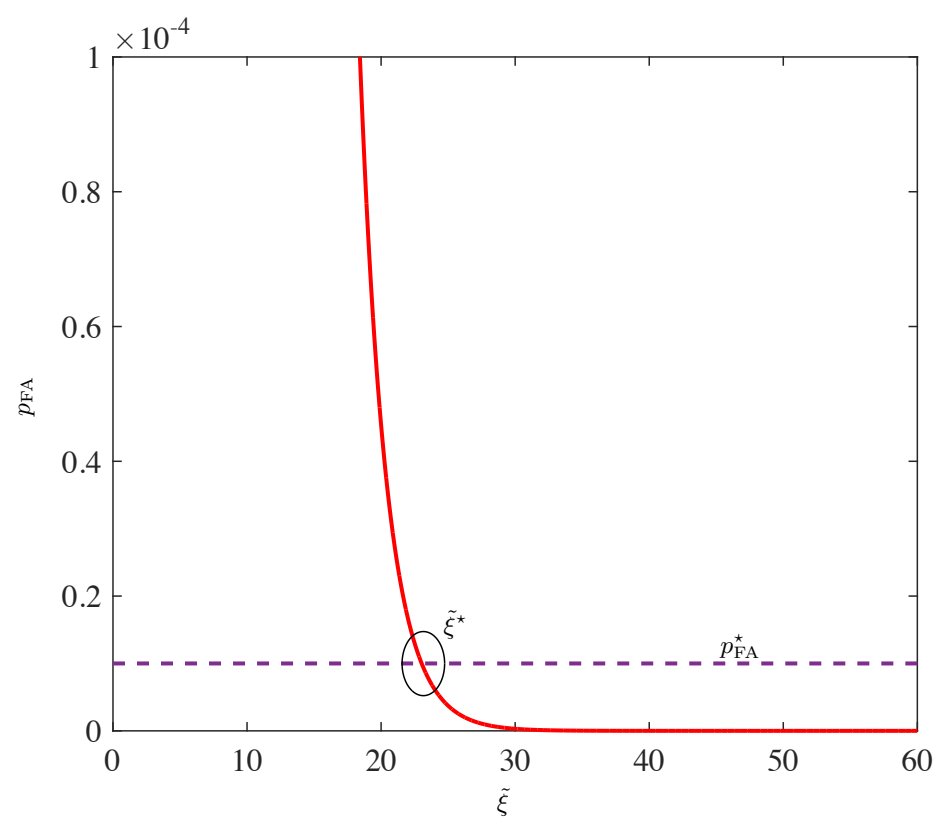

Fig. 3. Threshold choice in order to guarantee the target $p_{\mathrm{FA}}^{\star}$.

\section{Case Study}

We now consider the previously described system in order to evaluate what happens when real antennas are employed instead of ideal laser-beam antennas, which are accounted for the threshold design. Despite the analysis conducted is general, i.e. it can be applied to any frequency bandwidth, here we focus on mmW massive arrays, which could be one of the next fifth generation $(5 \mathrm{G})$ key technologies. This choice leads to an effective radiated isotropic power (EIRP) constrained by Federal Communications Commission (FCC) regulations as described in [15]. Thus, we first report threshold values in order to achieve a desired $P_{\text {FA }}^{\star}$ based on the receiver noise only. Once the threshold has been defined, we evaluate the bin-crossing probability for different values of the NCP, and finally we evaluate possible values in practical scenarios.

\subsection{Threshold Setting}

According to the analysis of Sec. 2, we now evaluate the threshold considering the receiver noise level. In particular, if otherwise indicated, we consider a time frame $T_{\mathrm{f}}=100 \mathrm{~ns}$, a bandwidth $W=1 \mathrm{GHz}$ and a time integration interval $T_{\mathrm{ED}}=1 \mathrm{~ns}^{2}$ In this way, by setting an overall $P_{\mathrm{FA}}^{\star}=10^{-3}$, it is $p_{\mathrm{FA}}^{\star}=10^{-5}$ which

\footnotetext{
${ }^{2}$ From [16], the threshold is accurate for large values of $N$, whereas for low $W T_{\mathrm{ED}}$ values, approximations could improve the accuracy of the threshold. Here we kept $N=2 W T_{\mathrm{ED}}$ since the effects do not affect the validity of the analysis.
} 


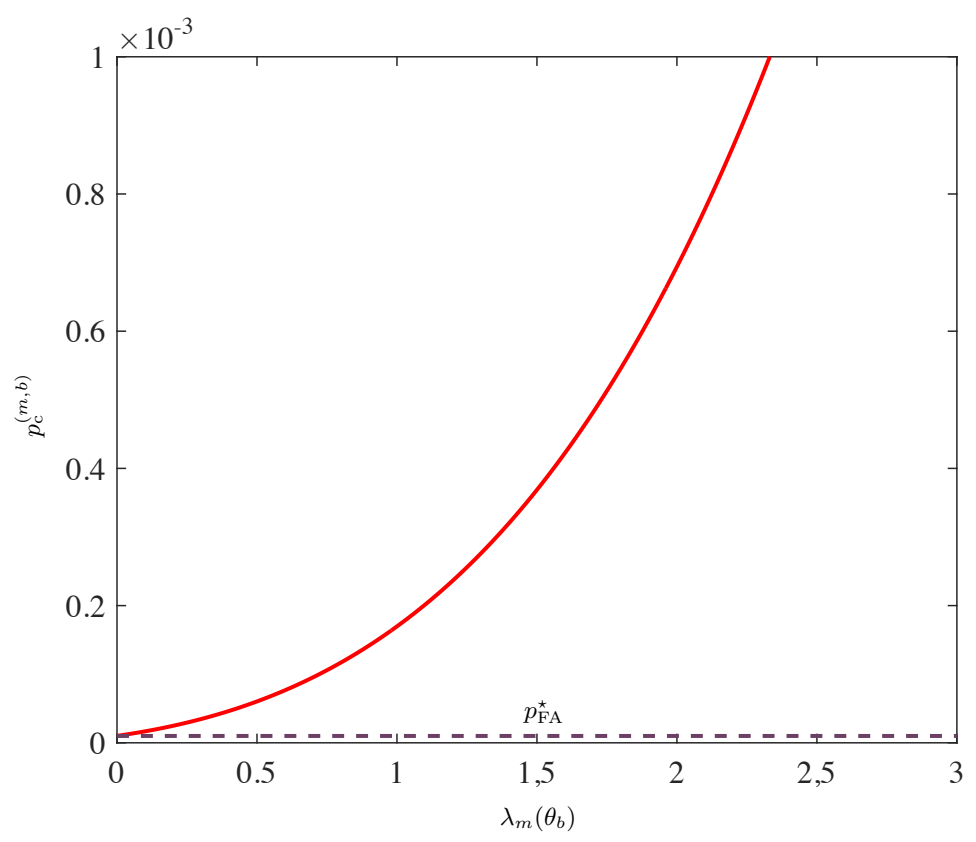

Fig. 4. Bin-crossing probability when the threshold $\tilde{\xi}^{\star}$ of Fig. 3 is adopted.

gives the threshold $\tilde{\xi}^{\star}$ reported in Fig. 3 . What it is important to remark is that such desired normalized threshold has been set according to the noise receiver only. Consequently, the impact of signals deriving from side-lobe directions has to be evaluated.

\subsection{Side-Lobes Effects}

When realistic antennas are adopted, and targets outside the steering direction are present, the threshold might be overcome. Consequently, such targets are wrongly associated with $\theta_{b}$, which translates into a possible detection and localization error. Thus, by considering (16), it is possible to estimate such effects when $\lambda_{m}\left(\theta_{b}\right)$ is greater than 0 .

In particular, we considered in (16), the $\xi^{\star}$ obtained in Fig. 3 in order to preserve an overall $P_{\mathrm{FA}}^{\star}=10^{-3}$. The obtained results are reported in Fig. 4, where it is evicenced that for $\lambda_{m}\left(\theta_{b}\right)<0.2$, the single bin $p_{\mathrm{c}}^{(m, b)}$ is still close to the desired value of $10^{-5}$. On the contrary, if we account for $\lambda_{m}\left(\theta_{b}\right) \approx 2.25$, it is even $p_{\mathrm{c}}=10^{-3}=P_{\mathrm{FA}}^{\star}$. Obviously, for such values of $\lambda_{m}\left(\theta_{b}\right)$, the system is not robust for target detection in $\theta_{b}$, as it is extremely sensitive to the presence of a target outside the desired direction.

In the following, we try to map generic values of the NCPs $\lambda_{m}\left(\theta_{b}\right)$ to those which can be obtained when massive arrays, as the ones described in [3], are adopted in practical applications. 
Table 1. SLL and maximum gain at $f_{\mathrm{c}}=60 \mathrm{GHz}$ for different phase compensation conditions.

\begin{tabular}{c|cc}
\multicolumn{2}{c}{$\theta_{b}=0^{\circ}$} \\
\hline Quantization & $G_{\max }[\mathrm{dBi}]$ & $\mathrm{SLL}[\mathrm{dB}]$ \\
& & \\
Perfect & 27.1 & 23.3 \\
3 bits & 26.9 & 24.6 \\
$\mathbf{2}$ bits & 26.4 & 20.6 \\
$\mathbf{1}$ bit & 23.5 & 16.8 \\
\hline
\end{tabular}

\begin{tabular}{c|cc}
\multicolumn{3}{c}{$\theta_{b}=20^{\circ}$} \\
\hline Quantization & $G_{\max }[\mathrm{dBi}]$ & SLL $[\mathrm{dB}]$ \\
& & \\
Perfect & 26.8 & 22.8 \\
3 bits & 26.5 & 20.7 \\
$\mathbf{2}$ bits & 25.9 & 19.4 \\
$\mathbf{1}$ bit & 22.2 & 13.6 \\
\hline
\end{tabular}

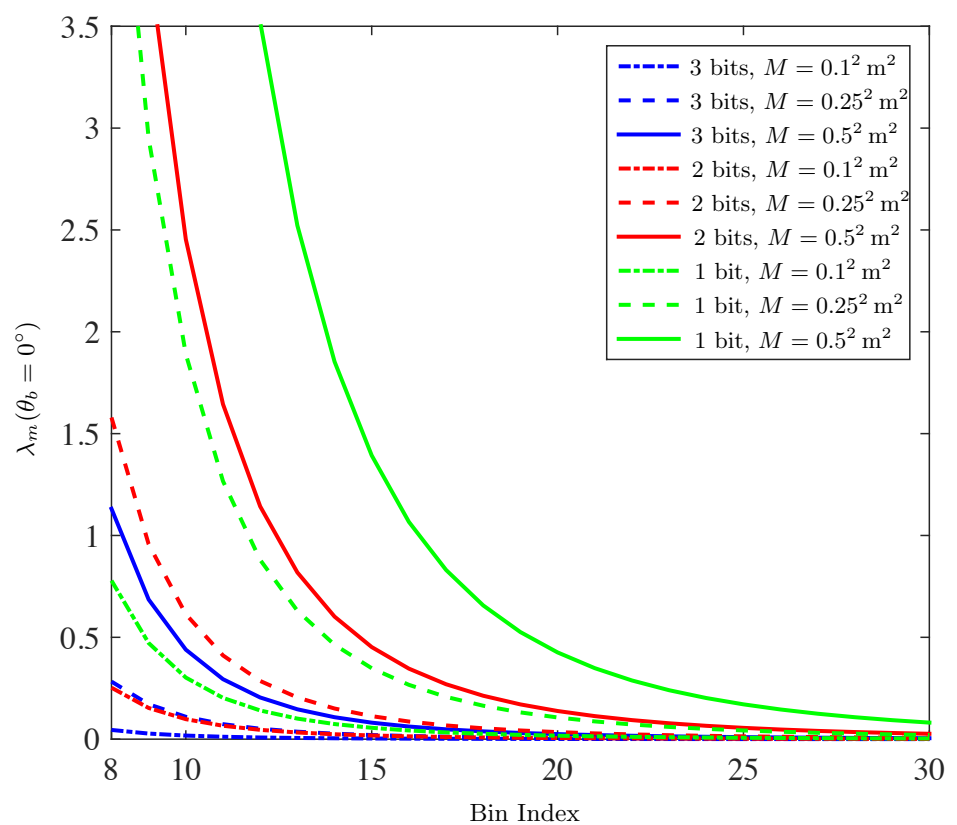

Fig. 5. NCP values for different values of $M$ and for $\theta_{b}=0^{\circ}$.

\subsection{Numerical Evaluation of the NCP}

Considering the previous results, we now map the obtained NCP $\lambda_{m}\left(\theta_{b}\right)$ into possible real values. In particular, we considered a receiver noise figure $F=4 \mathrm{~dB}$ and $T_{0}=290 \mathrm{~K}$. A simple and practical solution is to consider free-space propagation from the target to the radar section, and to assume the entire backscattered energy contained into one bin, which represents a worst case scenario. Successively, we dimension $\lambda_{m}\left(\theta_{b}\right)$ according to the expected path-loss of the signal in each bin from a side-lobe direction. We obtained

$$
\lambda_{m}\left(\theta_{b}\right)=2 \gamma_{m}\left(\theta_{b}\right)=\frac{1}{\sigma^{2}} \int_{W} S(f) \cdot G_{\mathrm{sl}}^{2}\left(f, \theta_{b}\right) M \frac{c^{2}}{f^{2}(4 \pi)^{3} d_{m}^{4}} d f
$$




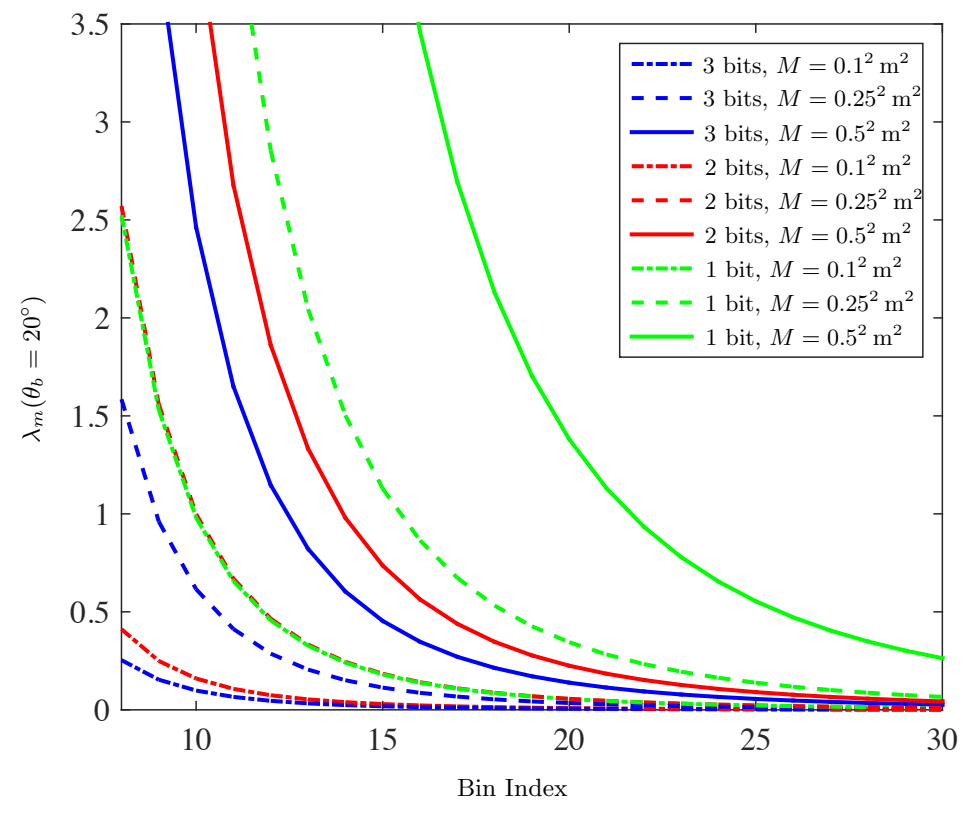

Fig. 6. NCP values for different values of $M$ and for $\theta_{b}=20^{\circ}$.

where $M$ is the target radar cross-section in the side-lobe direction, ${ }^{3} G_{\mathrm{sl}}\left(f, \theta_{b}\right)$ is the maximum side-lobe gain in the steering direction $\theta_{b}, S(f)$ is the transmitted power spectral density (PSD) and $d_{m}$ is the target-array distance. Note that the PSD has been set so that EIRP, evaluated according to $G_{\max }$, is compliant with the FCC regulations. In our scenario we fixed $\mathrm{EIRP}=30 \mathrm{dBm}$.

As existing antennas, we consider $15 \times 15$ massive arrays, which are a possible candidate for this kind of applications thanks to their narrow beam [3], by accounting for a different number of quantization bits which impact in the array pattern. As an example, Table 1 reports different values of $G_{\max }$ and $G_{\mathrm{sl}}$ at the central frequency $f_{\mathrm{c}}=60 \mathrm{GHz} .{ }^{4}$ The SLL represents the difference (in $[\mathrm{dB}])$ between the maximum gain and the peak of the main side lobe $G_{\mathrm{sl}}$.

In Fig. $5, \lambda_{m}\left(\theta_{b}\right)$ values are reported according to different quantization bits, the bin index (i.e. the target distance from the TX/RX) and different values of $M$. Consequently, according to Fig. 4 , we found that $\lambda_{m}\left(\theta_{b}\right)$ is often above 0.2 , which was found as a limit value in order to preserve $p_{\mathrm{c}}^{(m, b)}=p_{\mathrm{FA}}^{\star}$ in the presence of side-lobes. Note that the values of $\lambda_{m}\left(\theta_{b}\right)$ are also strictly related to the steering direction. In fact, when electronically beamsteering is performed, the SLL might increase, as also reported in Table 1 . Indeed, also the $\lambda_{m}\left(\theta_{b}\right)$ values change, as clearly evidenced in Fig. 6, especially when a low number

\footnotetext{
${ }^{3}$ Note that here we neglected the dependency of $M$ with the frequency.

4 The values account also for the spillover loss when massive arrays, such as transmitarrays [2], are excited with an external source.
} 
of quantization bits is adopted. This effect suggests that the SLL should be treated differently for each steering direction, as it is a key design parameter to be taken into account both for the massive array choice and for the threshold evaluation criterion. From one side, in order to reduce the interfering signal coming from directions different from $\theta_{b}$, it is important to reduce at most the SLL: for example, in our case study, at least 3 quantization bits are required to preserve reliable performance. On the other side, the choice of the massive array could be jointly performed with other operations in order to improve the performance. In fact, different techniques can be adopted in order to counteract side lobes effects on target detection. A possibility could be the adoption of a sidelobe blanker. In particular, a guard channel, which can be omni-directional or adaptive according to the direction, can be implemented to eliminate impulsive interference (hostile or from other neighboring radars) [17,18]. Analogously, in [19], a technique to mitigate the image artifacts due to the sidelobes of the random array is reported. All these solutions are appealing, but they can not be adopted due to their computational complexity and the use of coherent receivers.

A simple and effective solution could be the conception of new threshold design strategies, which accounts for both the receiver noise and the impact of the non-central parameters $\lambda_{m}\left(\theta_{b}\right)$. Future works will consider a CFAR approach where the $P_{\mathrm{FA}}^{\star}$ and the different level of interferers per bin are used to properly set the threshold.

\section{Conclusions}

In this paper we analyzed the impact of massive arrays side-lobes into detection performance for personal radars applications. In particular, in order to keep both the antenna array complexity and the cost low, a discrete set of phase shifts are often adopted for beamforming at the expense of an increased side-lobe level. In these situations, the design of a threshold accounting only for the receiver noise is not sufficient to guarantee the correct functioning of the system in terms of detection performance. In fact, as demonstrated by simulation results, the presence of side-lobes could drastically increase the crossing probability even when there is no target in the steering direction. This effect poses several attentions in the massive array choice according to its maximum SLL. Future studies will investigate the design of a threshold which accounts for the side-lobes effect in each steering direction.

\section{Acknowledgments}

This research was supported in part by the IF-EF Marie-Curie project MAPS (Grant 659067) and by the European H2020 project XCycle (Grant 635975). 


\section{References}

1. F. Guidi, A. Guerra, and D. Dardari, "Personal mobile radars with millimeter-wave massive arrays for indoor mapping," IEEE Trans. Mobile Comput., 2015.

2. A. Clemente, L. Dussopt, R. Sauleau, P. Potier, and P. Pouliguen, "Wideband 400-element electronically reconfigurable transmitarray in X band," IEEE Trans. Antennas Propag., vol. 61, no. 10, pp. 5017-5027, Oct 2013.

3. A. Guerra, F. Guidi, A. Clemente, R. D'Errico, L. Dussopt, and D. Dardari, "Application of transmitarray antennas for indoor mapping at millimeter-waves," in Proc. IEEE European Conf. on Networks and Commun. (EUCNC), 2015.

4. M. W. M. G. Dissanayake, P. Newman, S. Clark, H. Durrant-Whyte, and M. Csorba, "A solution to the simultaneous localization and map building (SLAM) problem," IEEE Trans. Robot. Autom., vol. 17, no. 3, pp. 229-241, Jun 2001.

5. E. Jose and M. Adams, "An augmented state SLAM formulation for multiple lineof-sight features with millimetre wave RADAR," in Proc. IEEE/RSJ Int. Conf. on Intelligent Robots and Syst., Aug 2005, pp. 3087-3092.

6. E. Jose, M. Adams, J. Mullane, and N. Patrikalakis, "Predicting Millimeter Wave Radar Spectra for Autonomous Navigation," IEEE Sensors J., vol. 10, no. 5, pp. 960-971, May 2010.

7. Y. Shen and M. Win, "Fundamental limits of wideband localization; part I: A general framework," IEEE Trans. Inf. Theory, vol. 56, no. 10, pp. 4956 -4980, oct. 2010.

8. L. Rondinelli, "Effects of random errors on the performance of antenna arrays of many elements," in IRE Int. Convention Record, vol. 7, March 1959, pp. 174-189.

9. J. Ruze, "Antenna tolerance theory - A review," Proc. IEEE, vol. 54, no. 4, pp. 633-640, April 1966.

10. H. Urkowitz, "Energy detection of unknown deterministic signals," Proc. IEEE, vol. 55, no. 4, pp. 523 - 531, april 1967.

11. M. Abramowitz and I. A. Stegun, Handbook of Mathematical Functions wih Formulas, Graphs, and Mathematical Tables. Washington, D.C.: United States Department of Commerce, 1970.

12. A. Mariani, A. Giorgetti, and M. Chiani, "Effects of noise power e imation on energy detection for cognitive radio applications," IEEE Trans. Commun., vol. 59, no. 12 , pp. $3410-3420$, december 2011.

13. M. Chiani, "Integral representation and bounds for marcum Q-function," IEEE Electronics Lett., vol. 35, no. 6, pp. 445-446, Mar. 1999.

14. F. Guidi, N. Decarli, S. Bartoletti, A. Conti, and D. Dardari, "Detection of multiple tags based on impulsive backscattered signals," IEEE Trans. Commun., vol. 62, no. 11, pp. 3918-3930, Nov 2014.

15. FCC, "Federal Communications Commission (FCC), 18-07-0082-01-0000d1, Amendment of Part 15 Rules for License-Exempt 57-64 GHz Band."

16. D. Slepian and E. Sonnenblick, "Eigenvalues associated with prolate spheroidal wave functions of zero order," Bell Sys. Tech. Journal, vol. 44, 1965.

17. U. Nickel, "Detection with adaptive arrays with irregular digital subarrays," in Proc. IEEE Radar Conf., April 2007, pp. 635-640.

18. C. Richmond, "Performance of the adaptive sidelobe blanker detection algorithm in homogeneous environments," IEEE Trans. Signal Process., vol. 48, no. 5, pp. 1235-1247, May 2000.

19. J. Tsao and B. Steinberg, "Reduction of sidelobe and speckle artifacts in microwave imaging: the CLEAN technique," IEEE Trans. Antennas Propag., vol. 36, no. 4, pp. 543-556, Apr 1988. 